\title{
Schief gewickelt?
}

\section{Moderne Therapie des Ulcus cruris}

Die Kompressionstherapie ist die Basis einer erfolgreichen Behandlung der meisten Patienten mit Ulcus cruris. Moderne Materialien und Systeme ermöglichen eine effektive und individualisierte Therapie.

Nicht nur Patienten mit einer chronischen venösen Insuffizienz, sondern nahezu alle Patienten mit Ulcus cruris und Ödemen profitieren von einer Kompressionstherapie, sofern strikte Kontraindikationen wie die fortgeschrittene $\mathrm{pAVK} / \mathrm{kritische}$ Ischämie $(\mathrm{ABPI}<0,5)$ ausgeschlossen wurden, er-

klärte Prof. Joachim

Dissemond, Universi-

tätsklinikum Essen im

Rahmen der 49. DDG-Ta-

gung in Berlin. „Die Rate derer, die eine Kompressionstherapie dringend benötigen würden - sie in der Regel aber dann nicht bekommen -, liegt sicherlich bei 80\%“. Trotz der sehr langen Tradition und der recht guten Evidenz der Kompressionstherapie, zeigten aktuelle wissenschaftliche Ergebnisse, dass die Versorgungsrealität in Deutschland unbefriedigend sei.

\section{„Niemals sollte sich der Patient selbst wickeln!"}

Auch Patienten mit Ulcus cruris mixtum könnten meist von der Kompressionstherapie profitieren, wie eine Untersuchung mit 25 Patienten zeigte, so Dissemond. Die Probanden hatten einen KnöchelArm-Druck-Index (ABPI) $>0,5$, absolute Druckwerte von mindestens $60 \mathrm{mmHg}$ und erhielten eine Kompression mit 20$50 \mathrm{mmHg}$ - alle gemessenen Parameter besserten sich bis $40 \mathrm{mmHg}$.

Die Kompressionstherapie kann heute mit sehr unterschiedlichen Materialien und Systemen durchgeführt werden. In Deutschland kommen meist Verbände mit Kurzzugbandagen zum Einsatz. Die Qualität dieser Verbände ist aber sehr stark von der jeweils anlegenden Person abhängig. „Niemals sollte sich der Patient selbst wickeln “, betonte Dissemond, dies
Von einer Kompressionstherapie können auch Patienten mit Ulcus cruris profitieren. sei fast immer ineffektiv. Auch Wundexperten könnten oftmals keinen "guten“ Kompressionsverband anlegen, viele wickelten zu locker.

\section{Adaptive Bandagen für die Entstauung}

Derzeit wenig genutzte Alternativen stellen Mehrkomponentensysteme und adaptive Bandagen dar, die insbesondere in der Entstauungsphase eingesetzt werden können. Bei den Mehrkomponentensystemen sollte man mit der „light“-Version beginnen (z. B. $20 \mathrm{mmHg}$ ). Marker auf den Kompressionsbinden, die bei korrekter Dehnung ein Symbol zeigen (z.B Oval wird zum Kreis), können das Anlegen eines effektiven Verbandes erleichtern. Bei adaptiven Systemen seien die Patienten in der Lage, den Druck meist selbst gut zu justieren - auch diese sollten anfangs auf $20 \mathrm{mmHg}$ eingestellt werden, riet Dissemond.

\section{Ulcus-Strumpfsysteme für die Erhaltungstherapie}

Für die Erhaltungstherapie sind es dann die Ulcus-Strumpfsysteme, die den Goldstandard der Kompressionstherapie des Ulcus cruris darstellen. Hier können auch zwei Strumpfsysteme kombiniert werden (Drücke addieren sich). Zur Rezidiv-Prophylaxe können medizinische Kompressionsstrümpfe eingesetzt werden.

Es bestehe Evidenz gemäß der Cochrane Collaboration für:

- Kompressionstherapie beschleunigt die Ulcus-Heilungsrate und reduziert die Rezidivrate bei Patienten mit Ulcus cruris venosum.

- Kompression mit Vierlagenverbandsystemen ist effektiver als andere Verbandsysteme mit Kurzzugbandagen.

- Zweilagenstrumpfsysteme sind effektiver als Kurzzugbandagen.

- Systeme mit mehreren Lagen sind effektiver als Systeme mit einem Verband.

- Vierlagenverbandsysteme sind kosteneffektiver als Verbandsysteme mit Kurzzugbandagen.

„Durch diese verschiedenen Behandlungsoptionen ist es heute möglich, für nahezu alle Patienten mit Ulcus cruris das passende System zu finden“, so Dissemond. Die individualisierte Kompressionstherapie sollte gemeinsam mit dem Patienten geplant und durchgeführt werden.

susanne Pickl

Symposium „Wunden", 49. Tagung der Deutschen Dermatologischen Gesellschaft (DDG),

26.04.-29.04.2017, Berlin 